\title{
An Alternative to Avrami Equation
}

\author{
José Roberto Costa Guimarães ${ }^{a}$, Paulo Rangel Rios ${ }^{a *}$, André Luiz Moraes Alves ${ }^{a}$ \\ ${ }^{a}$ Escola de Engenharia Industrial Metalúrgica de Volta Redonda, Universidade Federal Fluminense, Av. \\ dos Trabalhadores, 420, 27255-125, Volta Redonda, RJ, Brasil
}

Received: June 7, 2019; Revised: July 30, 2019; Accepted: August 8, 2019

\begin{abstract}
This paper proposes an alternative to the Avrami equation capable of describing whole transformation curves with significant fitting-correlations. The model bears physically meaningful parameters which permit considering the initial transformation kinetics independently from the subsequent microstructural evolution. Data of martensite, bainite, recrystallization, and pearlite transformations validate the model. Further to the expeditious description of transformation curves, the model guides the modeling of specific mechanisms.
\end{abstract}

Keywords: Phase transformations, Avrami's equation, martensitic transformation, bainitic transformation, pearlitic transformation, recrystallization.

\section{Introduction}

The description and the interpretation of transformation data are of considerable importance in research, in product/ process development, as well as in the production of engineering materials. However, one often has only partial or no understanding of the underlying mechanism of the transformation. In these circumstances, to describe the kinetic curve, i. e. fraction transformed against time, it is necessary to resort to phenomenological expressions.

The most used expression, in hundreds or thousands of papers, is the so-called Avrami equation ${ }^{1-4}$ :

$$
V_{V}=1-\exp \left(-k t^{n}\right)
$$

In Eq. (1), $V_{V}$ is the volume fraction transformed and $t$ is time. The constants $k$ and $n$ are fitting parameters. In the Avrami's equation, particular assumptions regarding nucleation and growth ${ }^{1-4}$ result in exact values of these constants. Moreover, the fitting parameters often admit interpretation in terms of the kinetic and microstructural aspects of the transformation.

Eq. (1) does possess certain limitations. For example, in Eq. (1) one has $V_{V}=0$ for $t=0$. That is, the transformation starts immediately. Nonetheless, in many transformations, one observes that there is an incubation time. The proposed alternative to Avrami's equation was introduced to describe martensitic transformations ${ }^{5-7}$. Subsequent work suggested that the equation could describe bainitic transformations? This work demonstrate the latter, as well as show that recrystallization and pearlite transformation also can be properly described and analyzed.

Notice that this work deals with iron alloys only. The Avrami's equation has found use in metallic glasses ${ }^{8}$, glasses $^{9}$, and polymers ${ }^{10}$. Thus one expects that Eq. (2). may be used to fit transformation curves in such systems.
Nonetheless, the utility of such fitting depends on the conceptualization of the model constants and parameters in terms of the intrinsic aspects of the transformation and/or processes.

\section{Theory}

The equation derived in the previous papers ${ }^{5-7}$ is:

$$
V_{V}=1-\exp \left(-V_{V i}\left(\frac{\xi-\xi^{*}}{\xi_{i}-\xi^{*}}\right)^{\varphi^{k}}\right)
$$

In Eq. (2) $\xi$ is an "advance" variable; is the fraction transformed for $\xi=\xi_{i}$. The constants $\xi^{*}$ and $\varphi^{\mathrm{K}}$ are fitting parameters.

In previous work ${ }^{5-7}, \xi$ was equal to temperature, magnetic field, mechanical deformation, and of course time. In this paper, one uses $\xi=t$. That is, this paper considers only isothermal kinetics.

$$
V_{V}=1-\exp \left(-V_{V i}\left(\frac{t-\tau}{t_{i}-\tau}\right)^{\phi^{K}}\right)
$$

In Eq. (3) $V_{V i}$ is the experimental fraction transformed at $t_{i}$. As above, $\tau$ and $\varphi^{\mathrm{K}}$ are fitting parameters. From Eq. (3) it is straightforward to determine the incubation time, $\tau$. After the incubation time the transformation proceeds. Therefore, it is natural to associate the other fitting parameter, $\varphi^{K}$, with the subsequent microstructural evolution. Of course, the microstructural evolution has a specific mechanism for each kind of transformation: martensite, bainite, recrystallization, or diffusional phase transformation. Therefore, one may expect $\varphi^{K}$ to mirror the different microstructural evolution mechanisms.

The similarity between Eq. (3) and Avrami's equation, Eq. (1) is notable. However, the advantage of Eq. (3) stems from its modular form ${ }^{11}$ that allows an analysis of the incubation time and $\varphi^{\mathrm{K}}$. Thus, Eq. (3) acknowledges the importance of the environment where the transformation occurs. 
The apparent activation energy for the initiation of the transformation is obtained from the temperature-dependence in $\tau$, drawing from classical kinetics,

$$
\frac{1}{\tau}=v P_{n} \exp \left(-\frac{E_{a}}{\mathrm{k}_{\mathrm{B}} T}\right)
$$

In Eq. (4) is a frequency, $P_{n}$ is the probability that the nucleation pathway is accessible to pre-nucleation assemblies, $E_{a}$ is an apparent activation-energy for such assemblies to overcome the nucleation barrier, $\mathrm{k}_{\mathrm{B}}$ is Boltzmann constant, and $T$ is the transformation temperature.

\section{Description of Experimental Transformation Curves by Eq. (3)}

The transformation curves of bainite in $\mathrm{Fe}-0.80 \mathrm{wt} \% \mathrm{C}$ $0.61 \mathrm{wt} \% \mathrm{Mn}-0.25 \mathrm{wt} \% \mathrm{~S}-0.2 \mathrm{wt} \% \mathrm{Ni}-0.2 \mathrm{wt} \% \mathrm{Cr}^{12}, \mathrm{Fe}-$ $0.99 w t \% \mathrm{C}-1.39 \mathrm{wt} \% \mathrm{Cr}-0.24 \mathrm{wt} \% \mathrm{Si}-0.29 \mathrm{wt} \% \mathrm{Mn}^{13}$, and $\mathrm{Fe}-0.29 \mathrm{wt} \% \mathrm{C}-2.39 \mathrm{wt} \% \mathrm{Mn}-1.76 \mathrm{wt} \% \mathrm{Si}^{14}$ can validate Eq. (3). The recrystallization curves typical of $\mathrm{Fe}-3.27 \mathrm{wt} \% \mathrm{Si}-$ $0.083 \mathrm{wt} \% \mathrm{C}^{15}$ and decarburized Ferrovac-E single crystal ${ }^{16}$ deformed by rolling can also validate Eq. (3). Finally, Eq. (3) could describe well a dataset on Pearlitic transformation of the Fe- $0.715 w t \% C-0.61 w t \% \mathrm{Mn}-0.347 \mathrm{wt} \% \mathrm{Si}-0.266 \mathrm{wt} \% \mathrm{Cr}$ steel ${ }^{17}$.

Scanning charts in the referenced papers generated these databases. A parametric least-squares procedure was used to fit the imported data. The imported data could not precisely determine the incubation time. Therefore, one considered the origin of the chart to be the first characterized datum $\left(V_{V i}, t_{i}\right)$ and expressed $\tau=\lambda \mathrm{t}_{i}$. Then the fitting parameter was $\lambda$.

The following figures show such fittings. Tables 1-3 depict the parameters of Eq. (3) for the bainite transformation. Tables 4 and 5 show the results of the recrystallization trials. Table 6 displays the pearlite transformation parameters.

\section{Isothermal Bainite Transformation}

The Fig. 1 and Table 1 refer to the Fe- $0.80 \mathrm{wt} \% \mathrm{C}$ bainite transformation curves described in ${ }^{12}$ with significant fitting correlations. The poor agreement of the $568 \mathrm{~K}$ transformation has been ascribed to the temperature dependence of the autocatalytic parameter in the original paper ${ }^{12}$.

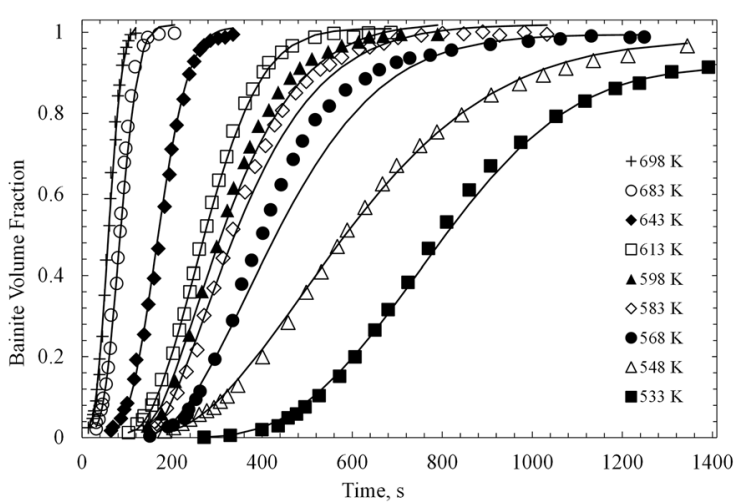

Figure 1. Fe- $0.80 \mathrm{wt} \% \mathrm{C}$ isothermal bainite transformation curves fitted with Eq. (3) - data from ref. ${ }^{12}$.

Observe in Table 1 that $\tau$ increases as the transformation temperature decreases. This increase in $\tau$ suggests a thermally activated kinetics. It is accepted that bainite nucleates heterogeneously, although the specific mechanism is still subject to controversy ${ }^{18}$. Nonetheless, it is reasonable to consider that pre-nucleation assemblies form and may access the nucleation pathway if thermal agitation does not impair their stability. The probability that of such nucleation event may be given by ${ }^{19}$,

$$
P_{n}(T)=\frac{T^{*}-T}{T}
$$

In Eq. (5) $T^{*}$ is the upper limit for the stability of such assemblies, and $T$ is the transformation temperature. The substitution of Eq. (5) into Eq. (4) yields the activation energy for the initial nucleation of bainite as a function of the transformation temperature

$$
E_{a}=\mathrm{k}_{\mathrm{B}} T \ln \left(v \tau \frac{T^{*}-T}{T}\right)
$$

In the present trial, the apparent activation energies for bainite nucleation, $E_{a}$, were calculated by inputting into Eq. (6) the values of from Table 1, $v=10^{13} \mathrm{~s}^{-1}$, $T^{*}=870 \mathrm{~K}^{20}$ and $\mathrm{k}_{\mathrm{B}}=8.310^{-3} \mathrm{~kJ} / \mathrm{mol}$. Note in Fig. 2 that the values of $E_{a}$ exhibit linear-variation on $\left(T^{*}-T\right)$ that is compatible with a dislocation mechanism ${ }^{21}$. Such values are compatible with energies reported in the literature, e.g., $160 \mathrm{~kJ} / \mathrm{mol}-165 \mathrm{~kJ} / \mathrm{mol}$ in ref. ${ }^{22}$.

Table 1. Isothermal bainite transformation in $\mathrm{Fe}-0.80 \mathrm{wt} \% \mathrm{C}$

\begin{tabular}{ccccc}
\hline$T, \mathrm{~K}$ & $\tau, \mathrm{s}$ & $\varphi^{\mathrm{K}}$ & $V_{V i}$ & $\mathrm{R}^{2}$ \\
\hline 698 & 13.82 & 2.40 & 0.025 & 0.98 \\
683 & 29.45 & 2.22 & 0.022 & 0.99 \\
643 & 62.95 & 2.38 & 0.018 & 0.99 \\
613 & 102.98 & 2.03 & 0.013 & 0.99 \\
598 & 145.65 & 1.51 & 0.029 & 0.99 \\
583 & 161.30 & 1.46 & 0.023 & 0.99 \\
568 & 150.50 & 1.78 & 0.010 & 0.98 \\
548 & 182.91 & 1.77 & 0.017 & 0.99 \\
533 & 269.72 & 2.55 & 0.001 & 0.99 \\
\hline
\end{tabular}




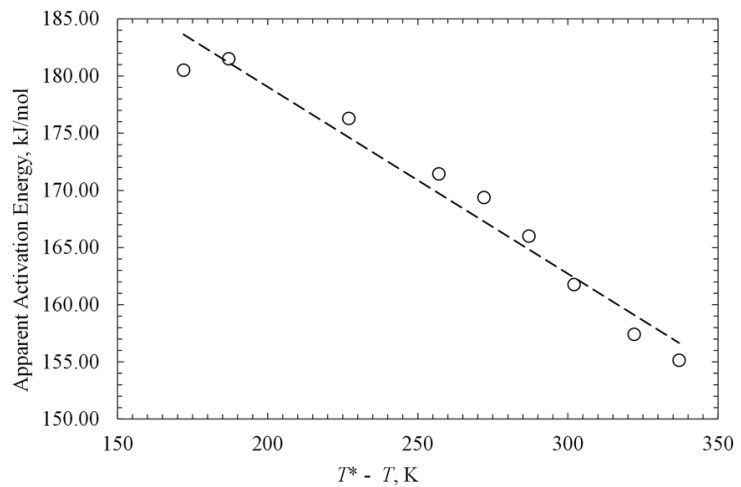

Figure 2. Fe- $0.80 \mathrm{wt} \% \mathrm{C}$ bainite: linear-variation of the activation energy for nucleation on $T^{*}-T$, where $T^{*}=870 \mathrm{~K}$.

Further inspection of Table 1 indicates that temperature dependence in the probability of intragrain transformation, $\varphi^{\mathrm{K}}$ is more complicated. Recalling Eq. (4)

$$
\varphi^{\mathrm{K}}=P_{i G} \exp \left(-\frac{Q_{i G}}{\mathrm{k}_{\mathrm{B}} T}\right)
$$

In Eq. (7) sub-iG refers to the intergrain transformation. Furthermore, assuming autocatalysis, one may set $P_{i G}(T) \cong 1$. Likewise, one may use an Arrhenius plot to characterize the variation in $\varphi^{\mathrm{K}}$ - see Fig. 3. Observe that the chart forks at an intermediate temperature. An apparent activation energy, $\sim 16 \mathrm{~kJ} / \mathrm{mol}$, one order of magnitude less than $E a$, characterizes the higher temperature branch. Such small energy is compatible with the excess activation energy for bainite nucleation in the presence of autocatalysis ${ }^{22}$. At the low transformation temperatures, anti-thermal $\varphi^{\mathrm{K}}$ is apparent. Notable, such a forked variation also was observed in the $\mathrm{Fe}-29.6 \mathrm{wt} \% \mathrm{Ni}$ isothermal transformation (lozenges). This behavior may relate to a change in the martensite substructure from lath into twinned plates ${ }^{6}$. This change is concurrent with the development of transformation chains driven by mechanical autocatalysis, which feeds back strain energy ${ }^{23}$. Thus, an anti-thermal variation in $\varphi^{\mathrm{K}}$ reiterates autocatalysis in bainite associated with the relaxation of transformation strains.

The fitting of the Fe- $0.99 w t \% \mathrm{C}-1.39 w t \% \mathrm{Cr}-0.24 \mathrm{wt} \% \mathrm{Si}-$ $0.29 \mathrm{wt} \% \mathrm{Mn}$ bainite transformation curves ${ }^{13}$ with Eq. (3) also was accomplished with significant fitting-correlations - see Fig. 4 and Table 2. As shown later, the variations in $\tau$ and $\varphi^{\mathrm{K}}$ fit the above considerations.

The incomplete Fe- $0.29 w t \% \mathrm{C}-2.39 \mathrm{wt} \% \mathrm{Mn}-1.76 \mathrm{wt} \% \mathrm{Si}$ bainite transformation curves in ref. ${ }^{14}$ also could be fitted with Eq. (3) with high fitting correlations - see Fig. 5.
In this case, Fig. 5 only show the fittings of three of the five imported datasets (lower, medium, and higher temperatures) to avoid cluttering. However, Table 3 lists the parameters of Eq. (3) pertinent to the whole database.

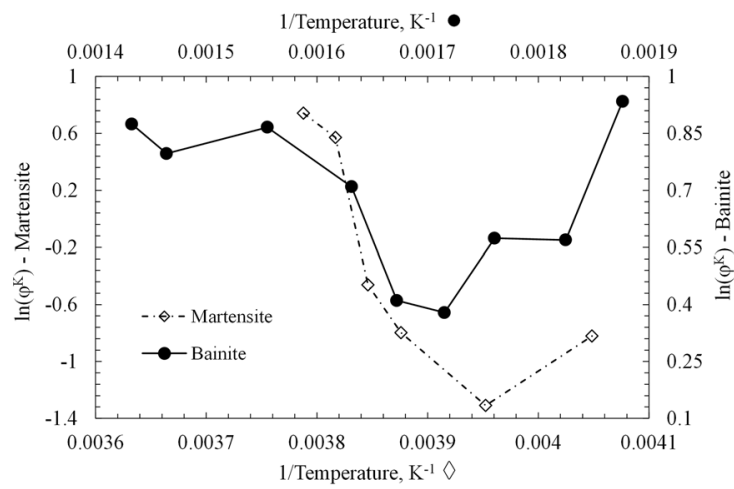

Figure 3. Temperature variation in $\varphi^{\mathrm{K}}$. Arrhenius plots: data typical of $\mathrm{Fe}-0.80 \mathrm{wt} \% \mathrm{C}$ bainite (circles) from Table 1, and $\mathrm{Fe}-29.6 \mathrm{wt} \% \mathrm{Ni}$ martensite (lozenges) from ${ }^{6}$.

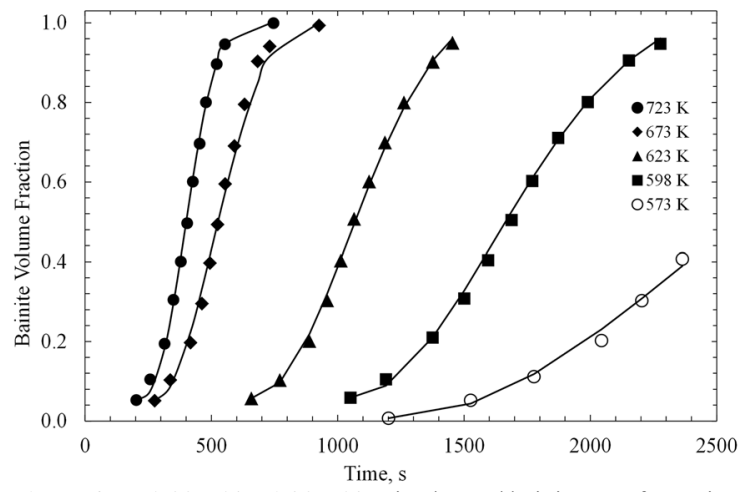

Figure 4. $\mathrm{Fe}-0.99 \mathrm{wt} \% \mathrm{C}-1.39 \mathrm{wt} \% \mathrm{Cr}$ isothermal bainite transformation curves fitted with Eq. (3) - data from ref. ${ }^{13}$.

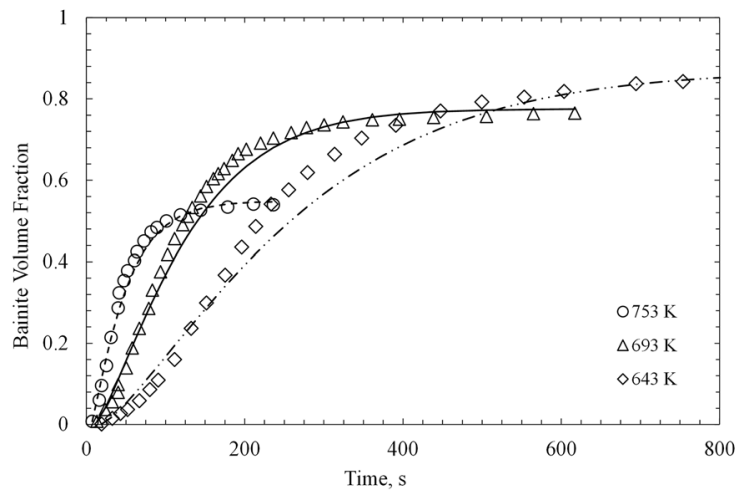

Figure 5. Fe- $0.29 w t \% \mathrm{C}-2.39 \mathrm{wt} \% \mathrm{Mn}-1.76 \mathrm{wt} \% \mathrm{Si}$ isothermal bainite transformation curves fitted with Eq. (3) - data from ref. ${ }^{14}$.

Table 2. Isothermal bainite transformation Fe- $0.99 \mathrm{wt} \% \mathrm{C}-1.39 \mathrm{wt} \% \mathrm{Cr}$

\begin{tabular}{ccccc}
\hline$T, \mathrm{~K}$ & $\tau, \mathrm{s}$ & $\varphi^{\mathrm{K}}$ & $V_{V_{i}}$ & $\mathrm{R}^{2}$ \\
\hline 723 & 200.55 & 2.62 & 0.053 & 0.99 \\
673 & 269.66 & 2.33 & 0.051 & 0.99 \\
623 & 652.78 & 2.11 & 0.057 & 0.99 \\
598 & 1048.95 & 1.99 & 0.069 & 0.99 \\
573 & 1198.23 & 2.09 & 0.007 & 0.98 \\
\hline
\end{tabular}


To consolidate the results of such validation trials, Figs. 6 and 7 show the activation energies as a function of temperature for $\tau$ and in $\varphi^{\mathrm{K}}$ typical of each steel. The former were calculated by inputting $v=10^{13} \mathrm{~s}^{-1}$ and $\mathrm{k}_{\mathrm{B}}=8.310^{-3} \mathrm{~kJ} /$ mol into Eq. (6). Considerations in the original papers ${ }^{13,14,20}$ helped to estimate $T^{*}$. Observe that in Fig. 6 activation energies of equal magnitudes are linearly related to the effective supercooling $\left(T^{*}-T\right)$. Thence, it is admissible that a similar mechanism operates the initial nucleation of the transformation, as might be expected. On the other hand, the $\mathrm{Fe}-0.29 \mathrm{wt} \% \mathrm{C}-2.39 \mathrm{wt} \% \mathrm{Mn}-1.76 \mathrm{wt} \% \mathrm{Si}$ bainite exhibited a distinct Arrhenius dependence in $\varphi^{\mathrm{K}}$. Mn-Si alloy possess smaller values of $\varphi^{\mathrm{K}}$ compared with Si-free steels. Both steels exhibited anti-thermal behavior whereas the other two steels thermal-activated behavior. Since anti-thermal $\varphi^{\mathrm{K}}$ refers to mechanical-autocatalysis, such counterpoint suggests that the feedback from auto-accommodation of the transformation strains did not compensate the drag effect imposed by Si influence on the carbon concentration in the austenite, thence the incomplete transformation as reasoned in ref. ${ }^{14}$.

\section{Recrystallization}

Eq. (3) fitted the database typical of the recrystallization in $60 \%$ deformed $\mathrm{Fe}-0.083 \mathrm{wt} \% \mathrm{C}-3.27 \mathrm{wt} \% \mathrm{Si}^{15}$ with significant fitting-correlations. The Fig. 8 demonstrate such fittings (dashed lines), where the signals refer to the datasets. Only four of those are shown to avoid cluttering. Table 4 depicts the parameters that characterize the fittings of the whole database.

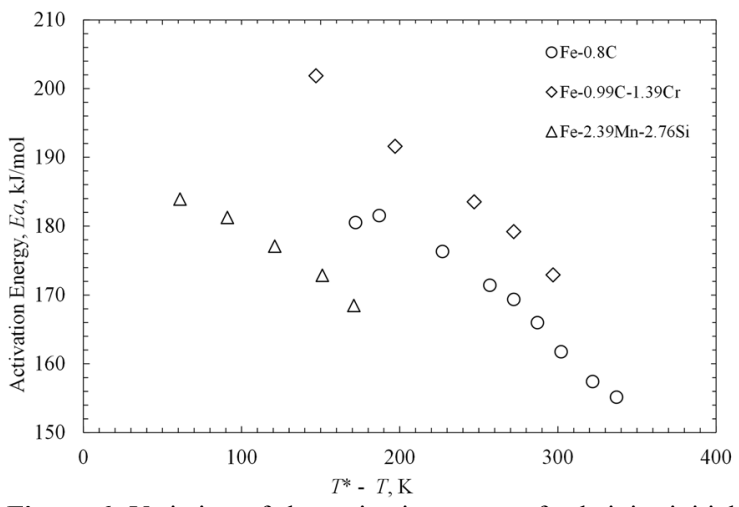

Figure 6. Variation of the activation energy for bainite initial nucleation in the different alloys as a function of the effective supercooling estimated by $T^{*}-T$.

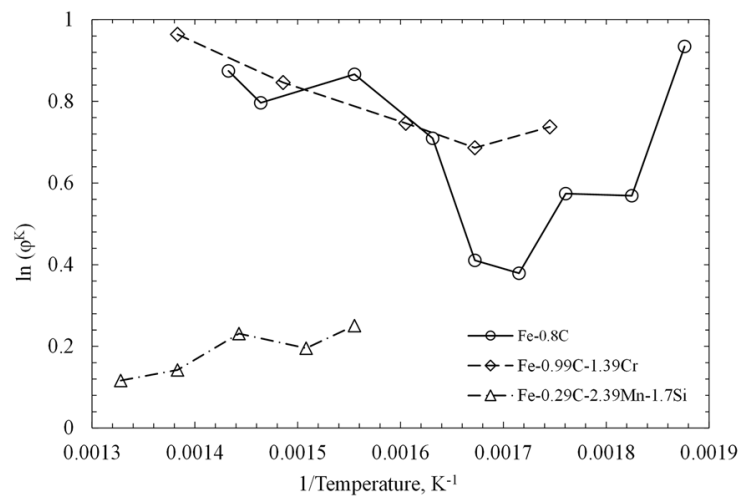

Figure 7. Arrhenius plots: variation in $\varphi^{\mathrm{K}}$ typical of bainite transformations in $\mathrm{Fe}-0.8 \mathrm{wt} \% \mathrm{C}, \mathrm{Fe}-0.99 \mathrm{wt} \% \mathrm{C}-1.39 \mathrm{wt} \% \mathrm{Cr}$, and $\mathrm{Fe}-0.29 \mathrm{wt} \% \mathrm{C}-2.39 \mathrm{wt} \% \mathrm{Mn}-1.7 \mathrm{wt} \% \mathrm{Si}$.

Table 3. Isothermal bainite transformation Fe- $0.29 \mathrm{wt} \% \mathrm{C}-2.39 \mathrm{wt} \% \mathrm{Mn}-1.76 \mathrm{wt} \% \mathrm{Si}$

\begin{tabular}{ccccc}
\hline$T, \mathrm{~K}$ & $\tau, \mathrm{s}$ & $\varphi^{\mathrm{K}}$ & $V_{V i}$ & $\mathrm{R}^{2}$ \\
\hline 753 & 7.43 & 1.12 & 0.009 & 0.98 \\
723 & 10.41 & 1.15 & 0.014 & 0.97 \\
693 & 13.38 & 1.26 & 0.008 & 0.98 \\
663 & 19.33 & 1.22 & 0.014 & 0.98 \\
643 & 19.33 & 1.29 & 0.001 & 0.98 \\
\hline
\end{tabular}

Table 4. Recrystallization: Fe-3.27wt $\% \mathrm{Si}-0.083 \mathrm{wt} \% \mathrm{C}$

\begin{tabular}{ccccc}
\hline$T, \mathrm{~K}$ & $\tau, \mathrm{s}$ & $\varphi^{\mathrm{K}}$ & $V_{V i}$ & $\mathrm{R}^{2}$ \\
\hline 1273 & 0.00198 & 0.59 & 0.069 & 0.99 \\
1225 & 0.00452 & 0.95 & 0.025 & 0.71 \\
1184 & 0.0148 & 1.42 & 0.008 & 0.76 \\
1123 & 0.0666 & 2.35 & 0.008 & 0.96 \\
1073 & 0.195 & 1.12 & 0.016 & 0.99 \\
1023 & 1.27 & 1.80 & 0.045 & 0.97 \\
973 & 5.13 & 1.21 & 0.026 & 0.79 \\
923 & 44.3 & 1.16 & 0.036 & 0.69 \\
873 & 395.00 & 1.10 & 0.016 & 0.96 \\
\end{tabular}




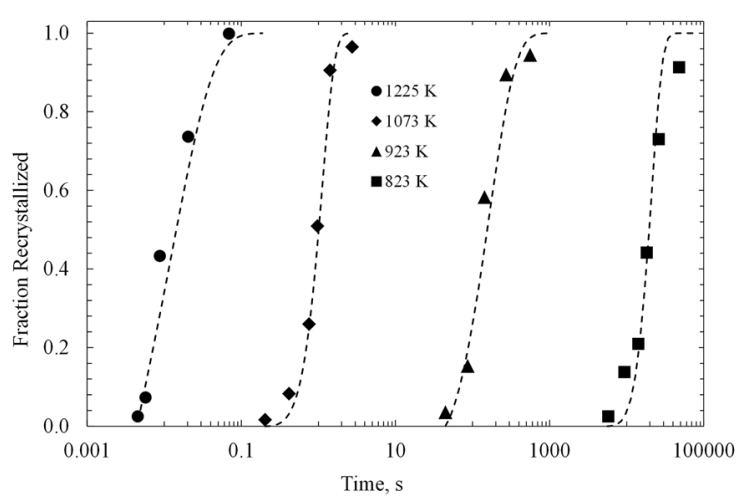

Figure 8. Fe-3.27wt $\%$ Si- $0.083 w t \%$ C: Data from ref. ${ }^{15}$ fitted with Eq. (3).

The chart in Fig. 9 characterizes the temperature dependence in the parameter $\tau$ based on Eq. (6), assuming that the strain energy resultant from the pre-deformation is frozen-in at $298 \mathrm{~K}$, $P_{n}(T) \cong \frac{T-298}{T}$, and using $v=10^{13} \mathrm{~s}^{-1}$ and $\mathrm{k}_{\mathrm{B}}=0.0083 \mathrm{~kJ} / \mathrm{mol}$. The obtained apparent activation-energy $Q_{R}=247 \mathrm{~kJ} / \mathrm{mol}$ fits in the interval between the $293 \mathrm{~kJ} / \mathrm{mol}$ reported in ref. ${ }^{15}$, and the 229.8 $\mathrm{kJ} / \mathrm{mol}$ typical of $50 \%$ cold-rolled Fe- $0.38 \mathrm{wt} \% \mathrm{C}-21.6 \mathrm{wt} \% \mathrm{Mn}^{24}$.

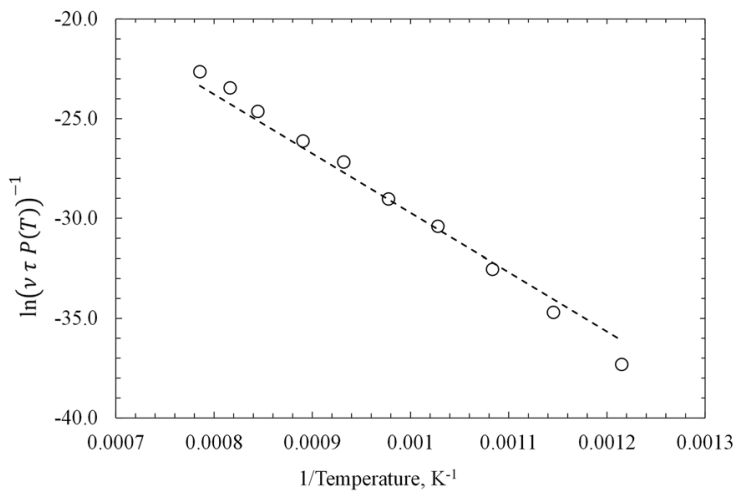

Figure 9. Fe-3.27wt $\% \mathrm{Si}-0.083 \mathrm{wt} \% \mathrm{C}$-Arrhenius plot. Characterization of the nucleation process - apparent activation energy: $247 \mathrm{~kJ} / \mathrm{mol}$.

The temperature dependence in $\varphi^{\mathrm{K}}$, Fig. 10, instead of a V-forked variation as observed in bainite and martensite, shows a conspicuous maximum at $\sim 1073 \mathrm{~K}$ which correlates with the variation of the mean recrystallized grain diameters reported $\mathrm{in}^{15}$, indicated by the lozenges. Considering the accepted knowledge about recrystallization, one refers $\varphi^{\mathrm{K}}$ to the probability of existing a flux of atoms from the deformed matrix into the recrystallized grains. To check this assertion, one calculates the probability that a local atom may cross the boundary between recrystallized and deformed material

$$
\varphi^{K}=\frac{\Delta}{\mathrm{k}_{\mathrm{B}} T} \exp \left(-\frac{Q m}{\mathrm{k}_{\mathrm{B}} T}\right)
$$

where the ratio $\frac{\Delta}{\mathrm{k}_{\mathrm{B}} T}$ gives the probability that a local atom accesses the recrystallization path, and $\exp \left(-\frac{Q_{m}}{\mathrm{k}_{\mathrm{B}} T}\right)$ refers to the probability that the atom crosses the recrystallization barrier.

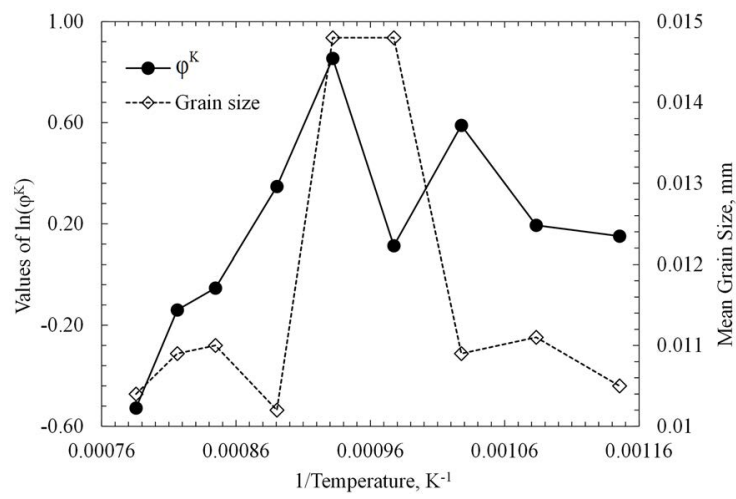

Figure 10. $\mathrm{Fe}-3.27 \mathrm{wt} \% \mathrm{Si}-0.083 \mathrm{wt} \% \mathrm{C}$ - Arrhenius - Variation of $\ln \left(\varphi^{\mathrm{K}}\right)$. The lozenges refer to the values of the mean recrystallized grain size tabulated in ref. ${ }^{15}$.

Assuming in Eq. (8) that neither $\Delta$ nor $Q_{m}$ exhibits significant temperature-dependence, one considers that $\varphi^{\mathrm{K}}$ varies with $\frac{\Delta}{\mathrm{k}_{\mathrm{B}} T}$ at high temperatures, and with $\exp \left(-\frac{Q_{m}}{\mathrm{k}_{\mathrm{B}} T}\right)$ at the lower temperatures. The energies so obtained are $\Delta=20 \mathrm{~kJ} / \mathrm{mol}$ and $\mathrm{Q}_{\mathrm{m}}=74 \mathrm{~kJ} / \mathrm{mol}$.

To compare, one uses the database typical of the recrystallization of single-crystalline decarburized Ferrovac-E described in ref. ${ }^{16}$. The dashed lines in Fig. 11 show the fittings of the imported database (signals). As in the previous cases, significant fitting-correlations were possible. The respective parameters in Eq. (3) are shown in Table 5.

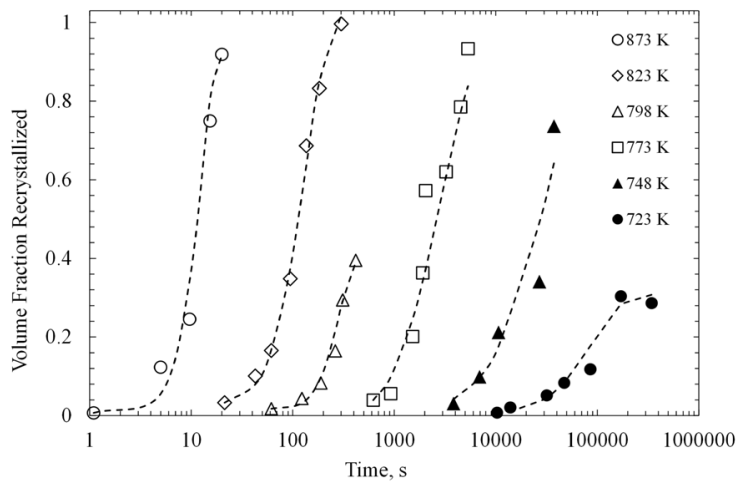

Figure 11. Decarburized Ferrovac-E: fitting of the data imported from ref. ${ }^{16}$ with Eq. (3).

Table 5. Recrystallization of single crystalline Ferrovac-E

\begin{tabular}{ccccc}
\hline$T, \mathrm{~K}$ & $\tau, \mathrm{s}$ & $\varphi^{\mathrm{K}}$ & $V_{V i}$ & $\mathrm{R}^{2}$ \\
\hline 873 & 0.00979 & 4.46 & 0.007 & 0.96 \\
823 & 21.20 & 1.78 & 0.033 & 0.99 \\
798 & 60.40 & 2.50 & 0.018 & 0.97 \\
773 & 620.00 & 1.24 & 0.039 & 0.94 \\
748 & 3820.00 & 1.18 & 0.031 & 0.89 \\
723 & 10300.00 & 1.48 & 0.008 & 0.95 \\
\hline
\end{tabular}


The analysis of $\tau^{-1}$ based on the Arrhenius equation, Fig. 12, yielded $Q_{R}=214 \mathrm{~kJ} / \mathrm{mol}$. Such energy bears the same magnitude but less than the $334 \mathrm{~kJ} / \mathrm{mol}(80 \mathrm{kcal} / \mathrm{mol})$ estimated by the time to reach $10 \%$ recrystallization reported in ${ }^{16}$.

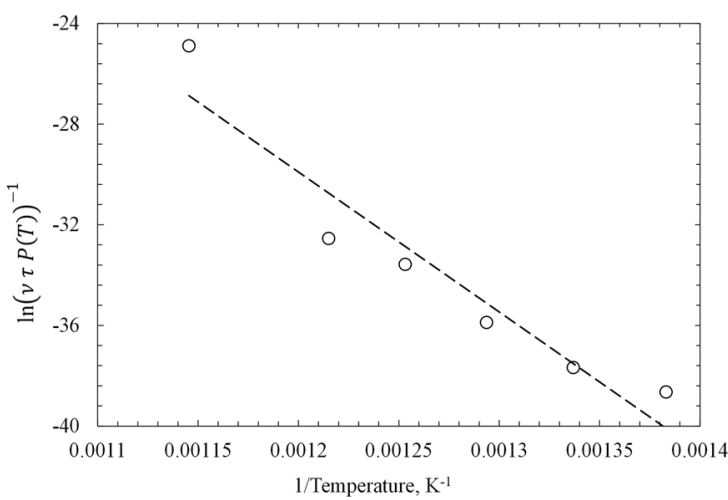

Figure 12. Determination of the apparent activation energy to initiate the recrystallization in decarburized single crystalline Ferrovac-E described in ref. ${ }^{16} Q_{R}=214 \mathrm{~kJ} / \mathrm{mol}$.

The temperature-dependence in $\varphi^{\mathrm{K}}$ does not show a maximum what may be attributed to insufficient stored energy in the deformed single crystal to sustain the driving forcecontrolled limb-see Fig. 13. Ref. ${ }^{16}$ mentions the possibility of a non-uniform distribution of the stored energy in the rolled single crystal. Noteworthy, the apparent activation energy, $31 \mathrm{~kJ} / \mathrm{mol}$, obtained from the chart in Fig. 13 bears the same magnitude as $Q_{m}=62 \mathrm{~kJ} / \mathrm{mol}$ obtained with the polycrystalline $\mathrm{Fe}-3.5 \mathrm{wt} \% \mathrm{Si}$.

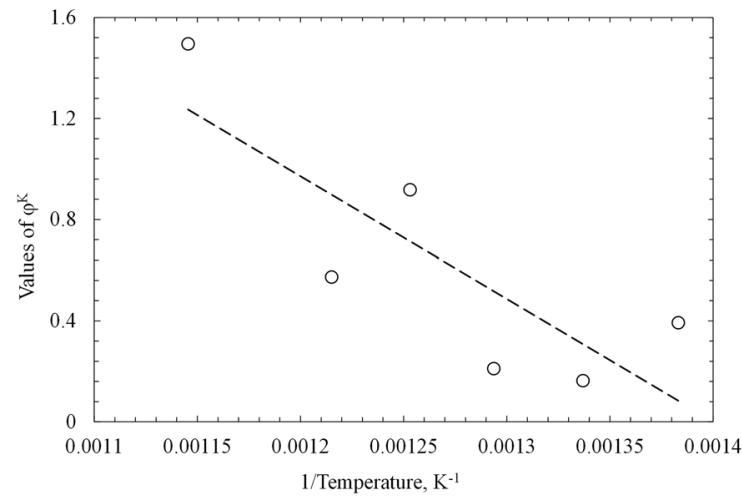

Figure 13. Values of $\varphi^{\mathrm{K}}$ typical of decarburized single crystalline Ferrovac-E described in ref. ${ }^{16} Q_{m X}=31 \mathrm{~kJ} / \mathrm{mol}$.

\section{Pearlitic Transformation}

The fitting of the Fe- $0.715 w t \% \mathrm{C}-0.61 \mathrm{wt} \% \mathrm{Mn}-0.347 \mathrm{wt} \% \mathrm{Si}-$ $0.266 \mathrm{wt} \% \mathrm{Cr}$ pearlite transformation curves described in ref. ${ }^{17}$ with Eq. (3) also was accomplished with significant fitting-correlations - see Fig. 14 and Table 6.

The limited scope of the database did not allow a discussion of the fitting parameters. However, it is apparent that the activation energies, $230 \mathrm{~kJ} / \mathrm{mol}-238 \mathrm{~kJ} / \mathrm{mol}$, obtained from the values of $\tau$, using $T^{*}=995 \mathrm{~K}, v=10^{13} \mathrm{~s}^{-1}$ and $\mathrm{k}_{\mathrm{B}}=$ $8.310^{-3} \mathrm{~kJ} / \mathrm{mol}$ into Eq. (6) are in the range of magnitude of the activation energy for boundary and volume diffusion of carbon reported in the literature e.g., refs. ${ }^{25,26}$. Additionally, a " $\Lambda$ trend" may be seen in the values of $\varphi^{\mathrm{K}}$ displayed in Table 6. On the other hand, the fitted charts displayed in Fig. 14 support Eq. (3) as an alternative to Avrami's equation.

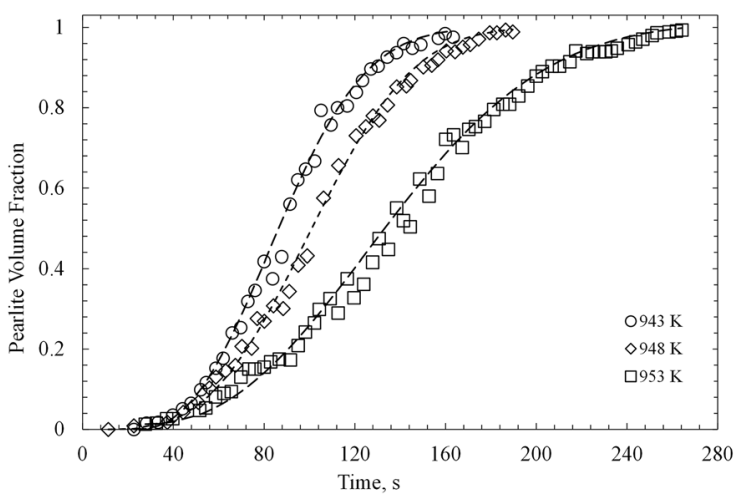

Figure 14. Fe- $0.715 \mathrm{wt} \% \mathrm{C}-0.61 \mathrm{wt} \% \mathrm{Mn}-0.347 \mathrm{wt} \% \mathrm{Si}-0.266 \mathrm{wt} \% \mathrm{Cr}$ isothermal pearlite transformation curves fitted with Eq. (3) - data from ref. ${ }^{17}$

\section{Conclusions}

Eq. (3), provides an alternative to the Avrami equation capable of describing whole transformation curves with significant fitting-correlations. The model consolidated into Eq. (3) bears physically meaningful parameters, the incubation time, $\tau$, and $\varphi^{\mathrm{K}}$ that refers to the kinetics of the microstructural evolution of the transformation. Thence, the model acknowledges distinct (local) reaction conditions.

The analyses of the incubation parameter, $\tau$, showed consistency with results in the literature. The parameter, $\varphi^{\mathrm{K}}$ which characterizes the microstructure evolution exhibits thermal-activated and anti-thermal regimes in either displacive or reconstructive transformations so that Eq. (7) may be used to consider a variety of transformation.

Table 6. Isothermal Perlite transformation Fe- $0.715 w t \% C-0.61 w t \% M n-0.347 w t \% S i-0.266 w t \% C r$

\begin{tabular}{ccccc}
\hline$T, \mathrm{~K}$ & $\tau, \mathrm{s}$ & $\varphi^{\mathrm{K}}$ & $V_{V_{i}}$ & $\mathrm{R}^{2}$ \\
\hline 953 & 27.70 & 2.30 & 0.000019 & 0.99 \\
948 & 11.30 & 2.94 & 0.000021 & 0.99 \\
943 & 22.60 & 2.44 & 0.000026 & 0.99 \\
\hline
\end{tabular}


Based on the present results, we contend that "V-shaped," variation in $\varphi^{\mathrm{K}}$ suggests nucleation-controlled transformation possibly assisted by autocatalysis, where a "-shaped" variation suggests growth-controlled transformation.

Consideration of the influence of driving force on the atomic mobility is crucial, e.g., in a reconstructive transformation such as recrystallization. At elevated temperatures, such bias may be impaired by thermal energy so that the factor $P_{n}=\frac{\Delta}{\mathrm{k}_{\mathrm{B}} T}$ controls the mobility of the boundary between the recrystallized and the deformed grains. At low temperatures $P_{n}$ is not an issue. However, the reduced thermal energy impairs the probability that atoms acquire the necessary potential energy to cross such boundaries. Thence thermally activated $\varphi^{\mathrm{K}}$ results, and the " $\Lambda$-fork" may be observed. In case the of displacive transformations, the analysis of the temperature dependence in $\varphi^{\mathrm{K}}$ point to the effect of the relaxation of transformation strains on the microstructure evolution. Here, at elevated temperatures, dislocation mobility fits purpose so that $\varphi^{\mathrm{K}}$ is thermally activated. Nonetheless, at low temperatures, slip processes may not suffice, so that complementary mutual-accommodation of transformation strains by variant selection sets-in, anti-thermal $\varphi^{\mathrm{K}}$ results, and one observes the "V-fork".

Summing up, the authors are aware that results extracted from a formal equation are model-dependent. Such an issue does not stand in the present analyses. This is so because characterizations of the initial transformation based on the parameter $\tau$ compares with results in the literature. Furthermore, the observed temperature-dependences in the parameter $\varphi^{\mathrm{K}}$ could be contextualized with differences between displacive and reconstructive aspects in the transformation data as well as with insights provided by the referenced papers. Of course, the model did not imply specific mechanisms but guides such developments. The fittings of transformation curves with Eq. (3) shows that the new model can describe transformation curves from incubation to saturation.

Therefore, the results of the validation trials warrant the utilization of the new model as a tool to describe experimental transformation curves as well as to characterize kinetic aspects of the transformation.

\section{Acknowledgments}

This study was financed in part by the Coordenação de Aperfeiçoamento de Pessoal de Nível Superior - Brasil (CAPES) - Finance Code 001. The authors are also grateful to Conselho Nacional de Desenvolvimento Científico e Tecnológico, CNPQ and Fundação de Amparo à Pesquisa do Estado do Rio de Janeiro, FAPERJ, for the financial support. Thanks are due to Professor Dr. H. Goldenstein, USP-SP, for his assistance with bibliography used in this paper.

\section{References}

1. Avrami M. Kinetics of phase change. I General Theory. The Journal of Chemical Physics. 1939;7(12):1103-1112.

2. Avrami M. Kinetics of phase change. II Transformation-Time relations for random distribution of nuclei. Journal of Chemical Physics. 1940;8(2):212-224

3. Avrami M. Granulation, phase change, and microstructure kinetics of phase change. III. The Journal of Chemical Physics. 1941;9(2):177-184

4. Barmak K. A Commentary on: "Reaction kinetics in processes of nucleation and growth". Metallurgical and Materials Transactions A. 2010;41A(11):1-65.

5. Guimarães JRC, Rios PR. Fundamental aspects of the martensite transformation curve in $\mathrm{Fe}-\mathrm{Ni}-\mathrm{X}$ and $\mathrm{Fe}-\mathrm{C}$ alloys. Journal of Materials Research and Technology. 2018;7(4):499-507.

6. Guimarães JRC, Rios PR. Revisiting temperature and magnetic effects on the Fe-30 Wt Pct Ni martensite transformation curve. Metallurgical and Materials Transactions A 2018;49(12):55956000 .

7. Guimarães JRC, Rios PR. General description of martensite transformation curves - a case for bainite. Materials Science and Technology. 2019;35(6):731-737.

8. Lu K. Nanocrystalline metals crystallized from amorphous solids: nanocrystallization, structure, and properties. Materials Science and Engineering. 1996;R16:161-221.

9. Málek J. The applicability of Johnson-Mehl-Avrami model in the thermal analysis of the crystallization kinetics of glasses. Thermochimica Acta. 1995;267:61-73.

10. Di Lorenzo ML, Silvestre C. Non-isothermal crystallization of polymers. Progress in Polymer Science. 1999;24(6):917-950.

11. Liu F, Sommer F, Bos C, Mittemeijer EJ. Analysis of solid state phase transformation kinetics: models and recipes. International Materials Reviews. 2007;52(4):193-212.

12. van Bohemen SMC. Autocatalytic nature of the bainitic transformation in steels: a new hypothesis. Philosophical Magazine. 2013;93(4):388-408.

13. Umemoto M, Horiuch K, Tamura I. Transformation kinetics of bainite during isothermal holding and continuous cooling. Tetsu-to-Hagane. 1982;68(3):461-470.

14. van Bohemen SMC, Hanlon DN. A physically based approach to model the incomplete bainitic transformation in high-Si steels. International Journal of Materials Research (formerly Zeitschrift für Metallkunde). 2012;103(8):987-991.

15. Speich GR, Fisher RM. Recrystallization of a rapidly heated 3.25 silicon steel. In: American Society for Metals. Recrystallization, Grain Growth, and Textures: Papers Presented at a Seminar of the American Society for Metals, October 16 and 17, 1965, Parte 1. Cleveland: ASM; 1966. p. 563-598.

16. Vandermeer RA, Rath BB. Modeling recrystallization kinetics in a deformed iron single crystal. Metallurgical Transactions A. 1989;20A(3):391-401. 
17. Offerman SE, van Wilderen LJGW, van Dijk NH, Sietsma J, Rekveldt MTh, van der Zwaag S. In-situ study of pearlite nucleation and growth during isothermal austenite decomposition in eutectoid steel. Acta Materialia. 2003;51:3927-3938.

18. Fielding LCD. The bainite controversy. Materials Science and Technology. 2013;29(4):383-399.

19. Guimarães JRC, Rios PR. Initial nucleation kinetics of martensite transformation. Journal of Materials Science. 2008;43(15):5206-5210.

20. van Bohemem SMC. Modeling Start Curves of Bainite Formation. Metallurgical and Materials Transactions $A$. 2010;41A:285-296.

21. Olson GB, Bhadeshia HKDH, Cohen M. Coupled diffusional/ displacive transformations. Acta Metallurgica. 1989;37:381-390.
22. Ravi A, Sietsma J, Santofimia MJ. Bainite formation kinetics in steels and the dynamic nature of the autocatalytic nucleation process. Scripta Materialia. 2017;140:82-86.

23. Bokros JC, Parker ER. The mechanism of the martensite burst transformation in Fe-Ni single crystals. Acta Metallurgica. 1963;11(12):1291-1301.

24. Lü Y, Molodov DA, Gottstein G. Recrystallization kinetics and microstructure evolution annealing of a cold-rolled Fe-Mn-C alloy. Acta Materialia. 2011;59:3229-3243.

25. Pandit AS. Theory of the Pearlite Transformation in Steels. Cambridge: University of Cambridge; 2012.

26. Seo SW, Jung GS, Lee JS, Bae CM, Bhadeshia HKDH, Suh DW. Pearlite growth rate in $\mathrm{Fe}-\mathrm{C}$ and $\mathrm{Fe}-\mathrm{Mn}-\mathrm{C}$ Steels. Materials Science and Technology. 2015;31:487-493. 